\title{
RIG-1 Expression Is Associated With Sexual Malfunctions of Female Type 2 Diabetic Patients
}

\author{
Batool Hajebrahimi ${ }^{1}$, Alireza Kiamanesh ${ }^{2 *}$, Ali Asghar Asgharnejad Farid ${ }^{3}$, Gholamreza Asadikaram ${ }^{4,5}$
}

${ }^{1}$ PhD Student in Psychology, Department of Psychology, School of Humanity and Social Sciences, Science and Research Branch, Islamic Azad University, Tehran, Iran

${ }^{2} \mathrm{PhD}$ in Research Methods and Evaluation, Department of Psychology, School of Humanity and Social Sciences, Science and Research Branch, Islamic Azad University, Tehran, Iran

${ }^{3} \mathrm{PhD}$ in Psychology, Mental Health Research Center, Department of Clinical Psychology, Tehran Institute of Psychiatry, Iran University of Medical Sciences, Tehran, Iran

${ }^{4} \mathrm{PhD}$ in Biochemistry, Neuroscience Research Center, Institute of Neuropharmacology, Kerman University of Medical

Sciences, Kerman, Iran

${ }^{5} \mathrm{PhD}$ in Biochemistry, Department of Biochemistry, School of Medicine, Kerman University of Medical Sciences, Kerman, Iran

*Correspondence to

Alireza Kiamanesh, Department of

Psychology, Faculty of Humanity,

Science and Research Branch,

Islamic Azad University, Tehran, Iran.

Tel: +982188072991

Email:

a.kiamanesh@srbiau.ac.ir

Received November 26, 2016 Accepted January 23, 2017

Published online February 15, 2017

\begin{abstract}
Introduction: The patients with type 2 diabetes (T2D) suffer from the malfunctions of the sexual behaviors, and several mechanisms have been proposed to describe these disorders. The innate immunity may be involved in the malfunctions of T2D patients. Melanoma differentiationassociated protein 5 (MDA5) and retinoic acid (RA)-inducible gene 1 (RIG-1), as the innate immunity receptors, are the responsible molecules for the activation of some intracellular signaling pathways and the induction of inflammation. Thus, this study aimed to examine the molecules which may participate in the induction/stimulation of sexual malfunctions in the female T2D patients.

Methods: Sexual functions were evaluated in 41 female T2D patients using the Female Sexual Function Index (FSFI) questionnaire. Real-time polymerase chain reaction (PCR) technique was used to quantify MDA5 and RIG-1 mRNA levels.

Results: Results showed that increased RIG-1 mRNA levels were significantly associated with the bad orgasm in the female T2D patients compared to the female patients with good orgasm. Expression of RIG-1 and MDA5 levels were not associated with other sexual functions' criteria. Conclusion: The findings of this study demonstrated that bad orgasm is associated with the increased RIG-1 expression. Consequently, the correlation between inflammation and bad orgasm in a RIG-1 dependent manner is suggested.

Keywords: Sexual function, Type 2 diabetes, Melanoma differentiation-associated protein 5, Retinoic acid-inducible gene 1.
\end{abstract}

\section{Introduction}

Sexual malfunctions are prevalent disorders in the humans, especially among the patients with immune related diseases. ${ }^{1}$ Accordingly, several studies have shown type 2 diabetic patients are main groups who are at risk of sexual malfunctions, especially female patients. ${ }^{1}$ Some investigations have approved the negative relations between sexual functions and inflammation. For instance, one study showed that a good sexual maturation suppresses the lung inflammation via the up-regulation of estrogen. ${ }^{2}$ Another study showed that sexual function's quality is dependent on the ovarian cycle related to inflammation. ${ }^{3}$ Thus, it seems that inflammation has a potential link with sexual functions, but specific molecules involved in inflammation affecting sexual functions are yet to be defined.

Innate immunity, as the keystone of the immune system, is activated via the interaction among its sensors, pattern recognition receptors (PRRs), and pathogens-associated molecular patterns (PAMPs)/ damage-associated molecular patterns (DAMPs). ${ }^{4-6}$ The PRRs are expressed either

Copyright $(2017$ The Author(s); Published by Zabol University of Medical Sciences. This is an open-access article distributed under the terms of the Creative Commons Attribution License (http://creativecommons.org/licenses/by/4.0), which permits unrestricted use, distribution, and reproduction in any medium, provided the original work is properly cited. 
on the cytoplasmic membrane or in the cytoplasm. Melanoma differentiation-associated protein 5 (MDA5) and retinoic acid(RA)-inducible gene 1 (RIG-1) are 2 key cytoplasmic receptors which play key roles in recognition of the viral mRNA and induction of the inflammation in the immune/non-immune cells via the PAMPs and DAMPs recognition and activation of 2 important transcription factors, interferon regulatory transcription factor 3 (IRF3) and nuclear factor-kappa B (NF-kB). ${ }^{7}$

Based on the potential correlation between inflammation and type 2 diabetes (T2D) ${ }^{6,8,9}$ together with high prevalence of sexual malfunctions in the patients, authors of this study hypothesized that alteration in MDA5 and RIG1 expressions may be associated with sexual malfunctions in the female T2D patients. Thus, this study aimed to assess the possible association between the MDA5 and RIG-1expression levels and sexual malfunctions in the female T2D patients.

\section{Methods}

Patients

In this cross-sectional study, T2D patients were diagnosed based on the American Diabetes Association Management Guidelines ${ }^{10}$ by an internal medicine doctor (MD). Accordingly, 41 female T2D patients, aged $54.09 \pm 2.71$ years old, were selected from Kerman Diabetic Clinical Center, Kerman University of Medical Sciences. Females suffering from T2D were included in the study. Blood samples were collected from them and the questionnaires were completed by them. The exclusion criteria were as follows: history of psychiatric disorders, existence of allergy, infectious diseases, cancers, autoimmune diseases, breastfeeding, pregnancy, and immunodeficiencies. Table 1 illustrates the demographic data of the participants. The patients' peripheral blood samples were collected in the tubes consisting anti-coagulant (ethylenediaminetetraacetic acid) for mRNA purification. The patients filled out the written informed consent forms as well as the Female Sexual Function Index (FSFI) questionnaires following the explanation of protocol and aims. Based on the questionnaires, the patients were divided into 2 bad and good sexual function groups. In addition, they were subdivided into other groups in terms of desire, orgasm, satisfaction, pain, arousal, and use of lubricant. The diagnosis of sex malfunctions was made by the psychiatrist. Accordingly, the experimental works including sampling, filling out the questionnaire, and gene analysis were performed in Kerman city.

Real-Time polymerase Chain Reaction RNA was purified from the whole peripheral blood immune cells using a commercial solution RNX (Catalog number [CN]: RN7713C) (Cinnaclon Company, Iran). Briefly, $200 \mu \mathrm{L}$ of blood was mixed with $200 \mu \mathrm{L}$ of RNX solution and after mixing; $50 \mu \mathrm{L}$ of chloroform was added and incubated at $4^{\circ} \mathrm{C}$ for 5 minutes. Then, the samples were centrifuged at $12000 \mathrm{rpm}$ for 8 minutes. The supernatant was transferred into a new RNase free tube and $500 \mu \mathrm{L}$ of $90 \%$ ethanol was added and mixed gently. After centrifugation at similar conditions, the supernatant was discarded and $70 \%$ ethanol was added to the tubes. After centrifugation at similar conditions, the supernatant was discarded and the tubes were incubated at room temperature to dry out the samples. Finally, 30 $\mu \mathrm{L}$ of RNase free water was added to resolve the purified RNA. The purified RNA was reversely transcribed to the cDNA using the Easy cDNA Synthesis kit (CN: A101162) (Pasteur Institute of Iran). In brief, the purified RNA and oligo-dT primers were incubated at $70^{\circ} \mathrm{C}$ and then cooled. The premix solution (containing reverse transcriptase enzyme, deoxynucleotides, and 95 suitable buffer) was added and incubated at $40^{\circ} \mathrm{C}$ for 60 minutes. The synthesized cDNA was amplified by real-time PCR using Exicycler ${ }^{\mathrm{TM}} 96$ Quantitative real-time PCR system and a commercial kit, $2 \mathrm{X} \mathrm{SYBR}^{\circledR}$ Green Real-Time PCR Master Mix (CN: C101021) from Pasteur Institute of Iran. The real-time PCR protocol was set as follows: 1 cycle; 5 minutes at $95^{\circ} \mathrm{C}, 40$ cycles; 40 seconds at $95^{\circ} \mathrm{C}$ and 45 seconds at $58^{\circ} \mathrm{C}$ and finally, extension: 45 seconds at $72^{\circ} \mathrm{C}$. $2-\Delta \Delta \mathrm{C}(\mathrm{T})$ formula was used to calculate the relative expression of the molecules under normalization of $\beta$-actin as the housekeeping gene. MDA5, RIG-1, and $\beta$-actin primer sequences were as follows:

$\beta$-actin Forward: GGCACCCAGCACAATGAAG, $\beta$-actin Reverse: CCGATCCACACGGAGTACTTG, RIG-1 Forward: CACACCAAGAGCCCAAAC, RIG-1 Reverse: TGACCCGATAGCAACAGC, MDA5 Forward: GCAGAGGTGAAGGAGCAGA, MDA5 Reverse: AAACGATGGAGAGGGCAAG.

\section{Data Analysis}

Calculation of data normality using the Kolmogorov-Smirnov test revealed that the data had a normal distribution. Thus, the differences between the patients with good and bad sexual functions were calculated using the parametric tests ( $t$ test). After the Levene test validation, $P$ value $<0.05$ was reported to be significant.

\section{Results}

The results revealed the mean expression of MDA5 mRNA levels in the female T2D patients with bad desire, arousal, lubrication, orgasm, satisfaction, pain and gen-

Table 1. Demographic Information of Participants ${ }^{a}$

\begin{tabular}{lccccc}
\hline Demographic Information & Age & Sex & FBS $(\mathbf{m g} / \mathbf{d L})$ & Hb1 Ac $(\%)$ & Duration of Diabetes $(\mathbf{y})$ \\
\hline Mean \pm SE & $54.09 \pm 2.71$ & Female & $188.62 \pm 15.06$ & $7.91 \pm 0.61$ & $11.65 \pm 1.87$ \\
\hline
\end{tabular}

Abbreviations: FBS, fasting blood sugar; Hb1Ac, hemoglobin A1c; SE, standard error.

a Table illustrates that the patients were female, and that the blood levels of FBS and Hb1Ac were higher than the normal values. 
erally sexual malfunctions were as $104.01 \pm 32.30,127.03$ $\pm 41.50,127.77 \pm 35.74,123.21 \pm 35.51,127.09 \pm 35.85$, $143.52 \pm 53.64$ and $127.77 \pm 35.74$, respectively (Figure 1). Data are illustrated as mean \pm standard error (SE). The mean expression levels of MDA5 in the T2D patients with good desire, arousal, lubrication, orgasm, satisfaction, pain and generally sexual malfunctions were $123.30 \pm$ $41.46,75.10 \pm 24.60,53.69 \pm 27.88,65.67 \pm 33.57,55.48$ $\pm 27.51,88.29 \pm 31.20$ and $53.69 \pm 27.88$, respectively (Figure 1). There were insignificant differences between bad and good desire ( $t:-261, d f: 27.00, P=0.796)$, arousal $(P=0.292, t: 1.076, d f: 25.662)$, lubrication $(t: 1.216, d f:$ 27.00, $P=0.234)$, orgasm ( $t: 0.935, d f: 27.00, P=0.358)$, satisfaction $(t: 1.174, d f: 27.00, P=0.251)$, pain $(t: 0.954$, $d f: 27.00, P=0.348)$ and generally $(t: 1.216, d f: 27.00$, $P=0.235)$ sexual malfunctions in the female T2D patients (Figure 1).

Expression of RIG-1 was increased significantly $(t: 2.128$, $d f: 29.532, P=0.042)$ in the female T2D patients with bad orgasm $(27.75 \pm 10.11)$ compared to the patients with good orgasm $(5.37 \pm 2.87)$. Expression of RIG-1 was not associated with bad and good desire $(t: 159, d f$ : $32, P=0.875)$, arousal $(t: 419, d f: 32, P=0.678)$, lubrica-
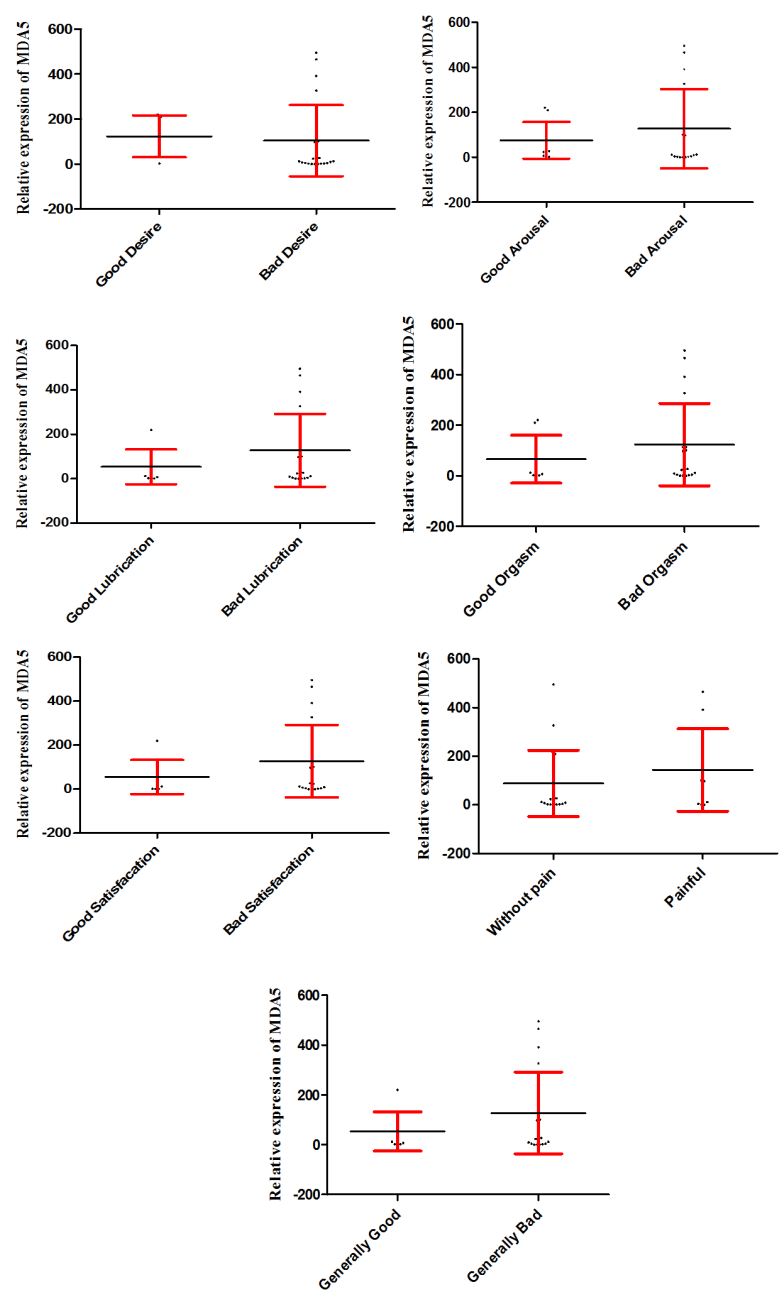

Figure 1. Relative Expression of MDA5 in the Female Type 2 Diabetic Patients in Various Occasions of Sex Functions. tion $(t: 480, d f: 32, P=0.634)$, satisfaction $(t: 0.480, d f: 32$, $P=0.635)$, pain $(t:-0.276, d f: 32, P=0.784)$ and generally ( $t: 0.323, d f: 32, P=0.749)$ sexual malfunctions in the female T2D patients (Figure 2).

\section{Discussion}

This study aimed to examine the correlation between the MDA5 and RIG-1 expression levels and sexual malfunctions in the female T2D patients. The results showed the RIG-1 expression increased significantly in the patients with bad orgasm, compared to the patients with good orgasm. Based on the fact that RIG-1, as an intracellular receptor, participates in the induction of inflammation, hence, it may be concluded that female T2D patients may suffer from bad orgasm because of induction/stimulation of inflammation in a RIG-1 dependent manner. Additionally, RIG-1 activates interferon regulatory factors (IRFs) which transcribes from type 1 interferons (IFN-Is). ${ }^{11}$ IFN-Is are important cytokines which interfere with brain functions in several disorders such as multiple sclerosis and Alzheimer. ${ }^{11}$ It is also proposed that the elevated levels of RIG-1 in the female T2D patients with bad orgasm is related to the increased expression of IFN-Is and their
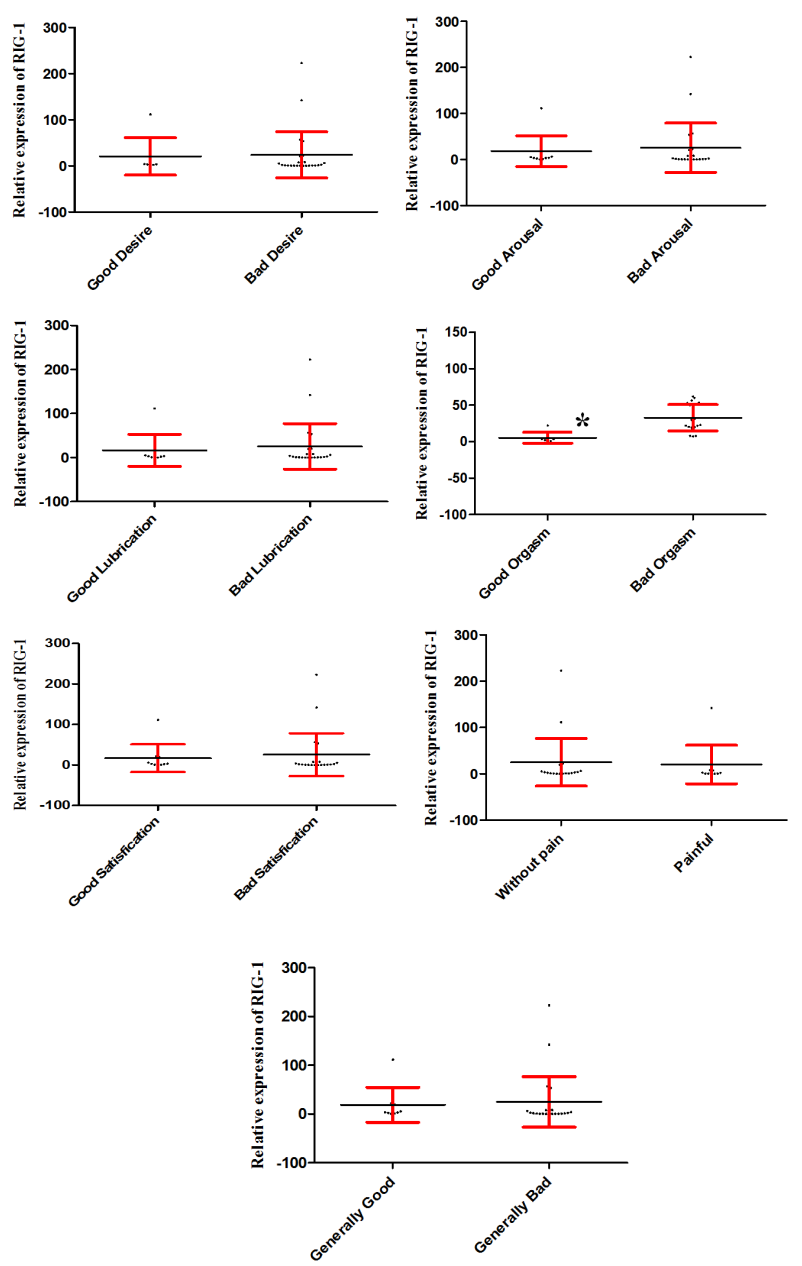

Figure 2. Relative Expression of RIG-1 in the Female Type 2 Diabetic Patients in Various Occasions of Sex Functions. 
effects on the brain functions.

There was no correlation between the expression of MDA5 and sexual functions of the female T2D patients, suggesting that MDA5 cannot be involved in the sexual malfunctions in the female T2D patients.

Several studies have demonstrated the role of inflammation in the induction of bad orgasm in the females. ${ }^{12}$ Additionally, mental disorders are the main causes of sexual malfunctions in the female T2D patients ${ }^{13,14}$; therefore, due to high prevalence of mental disorders in the patients, it has been hypothesized that the mental disorders are the main causes of sexual malfunctions in the female T2D patients.

Interestingly, the authors have already reported that mental disorders can be associated with the altered expressions of the innate immunity related molecules and inflammation induction. ${ }^{15,16}$. Thus, it may be hypothesized that increased expression of RIG-1 in the female T2D patients with the bad orgasm may be associated with mental disorders.

This study evaluated the correlation between the MDA5 and RIG-1expression levels and sexual malfunctions. However, the limitation of this study was small sample size. Hence, more investigations are required to improve our knowledge regarding the role of intracellular receptors such as MDA5 and RIG-1, on the sexual function. It is also suggested that future investigations focus on the RIG-1 as a target for the treatment of sexual malfunctions in the female $\mathrm{T} 2 \mathrm{D}$ patients.

\section{Conclusion}

This study showed that RIG-1 may participate in the induction of bad orgasm through the induction of inflammation in the female T2D patients. This study also suggested that RIG-1 may be involved in the induction of bad orgasm through other mechanisms.

\section{Ethical Approval}

The Ethical Committee of Kerman University of Medical Sciences evaluated and approved the study protocol with a code number of IR.KMU.REC1395.09.

\section{Competing Interests}

Authors declare that they have no competing interests.

\section{Acknowledgements}

This study was originated from a PhD thesis of the Science and Research Branch, Tehran Islamic Azad University, Iran. This project was supported financially by the Science and Research Branch, Tehran Islamic Azad University, Iran.

\section{References}

1. Elyasi F, Kashi Z, Tasfieh B, Bahar A, Khademloo M. Sexual dysfunction in women with type 2 diabetes mellitus. Iran J
Med Sci. 2015;40(3):206-213.

2. Draijer C, Hylkema MN, Boorsma CE, et al. Sexual maturation protects against development of lung inflammation through estrogen. Am J Physiol Lung Cell Mol Physiol. 2016;310(2):L166-L174. doi:10.1152/ ajplung.00119.2015

3. Lorenz TK, Worthman CM, Vitzthum VJ. Links among inflammation, sexual activity and ovulation Evolutionary trade-offs and clinical implications. Evol Med Public Health. 2015;2015(1):304-324.

4. Bagheri V, Askari A, Arababadi MK, Kennedy D. Can Toll-Like Receptor (TLR) 2 be considered as a new target for immunotherapy against hepatitis B infection? Hum Immun. 2014;75(6):549-554. doi:10.1093/emph/eov029. Print 2015.

5. Karimi-Googheri M, Arababadi MK. TLR3 plays significant roles against hepatitis B virus. Mol Biol Rep. 2014;41(5):3279-3286. doi:10.1007/s11033-014-3190-x.

6. Sepehri Z, Kiani Z, Nasiri AA, et al. Human Toll like receptor 4 gene expression of PBMCs in diabetes mellitus type 2 patients. Cell Mol Biol (Noisy-le-grand). 2015;61(3):92-95.

7. Ebrahim M, Mirzaei V, Bidaki R, et al. Are RIG-1 and MDA5 expressions associated with chronic HBV infection? Viral Immunol. 2015;28(9):504-508. doi:10.1089/vim.2015.0056

8. Sepehri Z, Kiani Z, Nasiri AA, Kohan F. Toll-like receptor 2 and type 2 diabetes. Cell Mol Biol Lett. 2016;21(1):2.

9. Andreasen AS, Pedersen-Skovsgaard T, Berg RM, et al. Type 2 diabetes mellitus is associated with impaired cytokine response and adhesion molecule expression in human endotoxemia. Intens Care Med. 2010;36(9):15481555.

10. Group NDD. Classification and diagnosis of diabetes mellitus and other categories of glucose intolerance. Diabetes. 1979;28(12):1039-1057.

11. de Rivero Vaccari JP, Brand FJ 3rd, Sedaghat C, Mash DC, Dietrich WD, Keane RW. RIG-1 receptor expression in the pathology of Alzheimer's disease. J Neuroinflammation. 2014;11(1):67. doi:10.1186/1742-2094-11-67.

12. Graziottin A, Gambini D, Vodusek D, Boller F. Anatomy and physiology of genital organs-Women. Handb Clin Neurol. 2015;130:39-60. doi: 10.1016/B978-0-444-632470.00004-3.

13. Shetageri VN, Bhogale GS, Patil NM, Nayak RB, Chate SS. Sexual dysfunction among females receiving psychotropic medication: a hospital-based cross-sectional study. Indian J Psychol Med. 2016;38(5):447-454.

14. Angel K. The history of 'Female Sexual Dysfunction' as a mental disorder in the 20th century. Curr Opin Psychiatry. 2010;23(6):536-541. doi:10.1097/YCO.0b013e32833db7a1.

15. Hajebrahimi B, Bagheri M, Hassanshahi G, et al. The adapter proteins of TLRs, TRIF and MYD88, are upregulated in depressed individuals. Int J Psychiatry Clin Pract. 2014;18(1):41-44. doi:10.3109/13651501.2013.85970 8.

16. Nazari M, Khodadadi H, Fathalizadeh J, et al. Defective NF$\mathrm{kB}$ transcription factor as the mediator of inflammatory responses: a study on depressed Iranian medical students. Clin Lab. 2013;59(7-8):827-830. doi:10.7754/Clin. Lab.2012.120809. 